\title{
Mikrochemischer Nachweis des Goldes mittels kolloidaler Färbung der Seidenfaser
}

\author{
von
}

\section{Julius Donau.}

Aus dem Laboratorium für allgemeine Chemie an der $k$. $k$. technischen Hochschule in Graz.

(Vorgelegt in der Sitzung am 17. März 1904.)

In den Arbeiten »Mikrochemischer Nachweis von Alkalien und Säuren " ${ }^{1}$ und "Notizen über die Lackmusseide " ${ }^{2}$ hat Prof. Emich sich der mit Lackmus gefärbten Seidenfaser bedient, um an deren Veränderungen äußerst geringe Mengen von Säuren und Alkalien zu entdecken und angedeutet, daß man auch andere Reaktionen heranziehen könnte, deren Äußerungen am Seidenfaden oder einer anderen Faser zur Auffindung geringer Mengen von Stoffen dienen würden.

In dieser Hinsicht zogen die Färbungen, welche Zsigmondy ${ }^{3}$, Henrich ${ }^{4}$ und andere bei der Reduktion von Goldlösungen durch gewisse Substanzen erzielten, in erster Linie die Aufmerksamkeit auf sich; außerdem war auch der Umstand maßgebend, daß bis jetzt keine sehr empfindlichen mikrochemischen Goldreaktionen bekannt zu sein scheinen. ${ }^{5}$ Eine solche auf dem angegebenen Weg zu suchen, war der $Z$ weck vorliegender Arbeit.

1 Monatshefte für Chemie, XXII, 670 .

2 Monatshefte für Chemie, XXIII, 76.

3 Annalen der Chemie, 301. B 29 bis 54 .

4 Berl. Ber., 36, 609 bis 616.

5 Über den mikrochemischen Nachweis des Goldes vergl. Behrens Anleitung zur mikrochemischen Analyse. 2. Aufl., 1899. 
Bevor indes auf dieses Thema übergegangen wird, soll eingeschaltet werden, daß sich purpurne Lösungen des Goldes z. B. auch herstellen ließen, wenn man eine verdünnte wässerige oder angesäuerte Lösung von Goldchloridchlorwasserstoff in ein Gefäß brachte, das vorher mit einer wässerigen Tanninlösung ausgespült worden war; die Purpurfärbung trat bei gewöhnlicher Temperatur langsam, beim Erwärmen rasch ein und ging geradeso wie die Zsigmondysche bei Zusatz eines Neutralsalzes, z. B. Kochsalz, sofort in blau über, wobei gleichzeitig Gold gefällt wurde. Bei Anw̄endung größerer Mengen des Reduktionsmittels wurde die Goldlösung nicht rot, sondern grünlich bis bläulich, ebenfalls unter gleichzeitiger Fällung des Goldes. Ebenso erhielt man in angesäuerter oder wässeriger Goldlösung Purpurfärbung bei Zusatz eines verdünnten Lösungsgemisches von Zinnchlorür und Pyrogallol und nachherigem Erwärmen.

Es gelang auch, die Boraxperle durch Gold charakteristisch zu färben; die Blase wurde zuerst mit verdünnter Goldlösung benetzt und hierauf in der Oxydations- oder Reduktionsflamme der Bunsenflamme zur Perle verschmolzen; diese zeigte sich auch bei Anwendung sehr geringer Goldmengen stets schön rubinrot, wurde aber nach längerem Erhitzen blau und endlich farblos bei Anwesenheit geringer Goldmengen, sonst leberfarbig. Diese Reaktion ist vielleicht zum qualitativen Nachweis des Goldes geeignet. ${ }^{1}$

Die Tatsache, daß sich Wolle, Leinwand, Haare, Seide und dergleichen, die mit Goldlösung in Berührung waren, am Lichte bald purpurn färben, ist längst bekannt. Tompa hat sie jüngst zu botanischen $Z$ wecken verwertet und angegeben, daß die Faserstoffe schon von Natur aus die Fähigkeit besitzen, Gold auf sich niederzuschlagen; er begründet diese Eigen-

1 Auf gleiche Weise gelang es, die Boraxperle auch durch andere Metalle zu färben; so färbten z. B. Platin die Perle rehbraun, Silber gelb mit starker Opaleszenz, Kadmium und Blei, beide nach Zusatz von etwas Zinkpulver gelb (nach dem Erkalten farblos). Offenbar handelt es sich auch hier um kolloidale Metallösungen. Näheres wird demnächst mitgeteilt werden.

2 Zeitschrift für wissenschaftliche Mikroskopie 20, p. 24 bis 28. 
schaft mit einem Gehalte der Fasern an Gerbsäure. Übrigens war vorliegende Arbeit schon im Gange, als Tompa's Mitteilung erschien.

Im folgenden wird gezeigt, wie die erwähnte Eigenschaft der Farbstoffe zu mikrochemischen Untersuchungen verwertet werden kann, und wie es damit gelingt, die Empfindlichkeitsgrenze der Goldreaktionen bedeutend zu erweitern.

\section{Behandlung der Faser.}

Es wurden vorläufig nur Seiden- und Baumwollfasern in Betracht gezogen. Bezüglich der Seidenfaser zeigte es sich, daf weder ganz rohe, noch eine durch lange Behandlung mit absolutem Alkohol und Äther peinlich gereinigte Seide besonders gute Resultate lieferte. Am wirksamsten schienen Fasern zu sein, die man aus Rohseide durch drei- bis vierstündige Behandlung mit etwa zehnprozentiger Kali- oder Natronlauge und nachheriges gründliches Auswaschen erhielt. Die ganz rohe Seide eignete sich schon deshalb nicht gut zur mikroskopischen Untersuchung, weil sich die einzelnen Fäden schwer trennen ließen, auch schien die große Menge Seidenleim, namentlich was die später $\mathrm{zu}$ beschreibende Purpurfärbung anbelangt, störend $z u$ wirken. Daß eine sehr reine Seide die Rotfärbung schlechter hervorbrachte, findet vermutlich darin eine Erklärung, daß eine solche Seide infolge ihrer Behandlung mit Alkohol und Äther sehr arm an einem wirksamen Kolloid geworden. ${ }^{1}$ Die Baumwolle wurde z. B. in Form von Bruns' Watte verwendet, zeigte sich aber weniger brauchbar als die oben erwähnte Seidenfaser. Es konnten auf der Baumwolle nur schwärzliche, bei Gegenwart von Salzen in schwach alkalischer Lösung blaue bis violette, seltener rote Färbungen erzielt werden.

Die Vorbereitung der Fasern bestand darin, daß dieselben längere Zeit in der Lösung oder dem Lösungsgemisch verschiedener Reduktionsmittel liegen mußten. Von den letzteren kamen in Betracht: Sulfite, Nitrite, Zinnchlorür, Oxalsäure, Formaldehyd, Hydrochinon und ein Hydrochinon-Entwickler, Tannin,

1 Zeitschrift für analyt. Chemie, 40. Jahrg, 11. Heft, p. 697. 
Pyrogallol, Zucker u. dgl. Von diesen erwiesen sich nur das Tannin und ein Gemisch von Zinnchlorür und Pyrogallol verwendbar. Das Tannin eignete sich sehr gut zur Vorbehandlung der Baumwolle, welche dann dunkel, beziehungsweise blau gefärbt wurde. Mit Tannin behandelte Seide wurde in der Goldlösung dunkel oder färbte sich gar nicht, falls diese Lösung alkalisch reagierte. Das Gemisch von Zinnchlorür und Pyrogallol ließ Wolle blau bis violett, in sehr verdünnter Goldlösung bisweilen rötlich, Seide hingegen stets rot werden.

Das Tannin (gew. Handelsprodukt) wurde in verdünnter wässeriger Lösung verwendet. Zinnchlorür wurde durch Auflösen von Stanniol in konzentrierter Salzsäure dargestellt. In die noch etwas freie Salzsäure enthaltende verdünnte Lösung wurde dann ein wenig Pyrogallol gebracht.

Vor dem Gebrauche wurde die Faser aus der Flüssigkeit genommen, einige Male durch Wasser gezogen und zwischen Filtrierpapier getrocknet. In diesem Zustande bewahrten die Fasern ihre Eigenschaft durch längere Zeit, z. B 2 bis 3 Tage; Fasern, die länger der Luft ausgesetzt waren, wurden in der Goldlösung dunkelfärbig.

Keine der erwähnten Färbungen konnte durch Säuren oder Basen aufgehoben werden. Nur Chloroder Bromwasser brachten sie zum Verschwinden, ein Umstand der unzweideutig auf ihre Goldnatur hinweist. Auch scheint es keinem $Z$ weifel $z u$ unterliegen, daß der rotgefärbte Seidenfaden diese Färbung kolloidal gelöstem Golde verdankt, ${ }^{1}$ während der Blau- und Dunkelfärbung der Fasern feinere oder gröbere Suspensionen von Goldteilchen zu Grunde liegen.

\section{Bereitung der Groldlösung.}

Am brauchbarsten, namentlich für die Rotfärbung wesentlich notwendig, erwies sich eine wässerige oder angesäuerte

\footnotetext{
1 Auch die später erwähnte Gelbfärbung durch Silbersalze spricht dafür; bekanntlich kann auch das Glas durch kolloidales Gold und Silber gefärbt werden.
} 
Lösung von Goldchloridchlorwasserstoff. Zur Darsteilung dieses Salzes wurde das Gold am Wasserbade in viel Königswasser aufgelöst oder mit chlorhältiger Salzsäure behandelt, in welche man auch nach dem Verschwinden des Metalls noch eine zeitlang Chlor einleitete. Die Flüssigkeit wurde eingedampft und in Wasser aufgenommen. Durch eine solche Lösung konnte die erwähnte Rotfärbung des Seidenfadens sicher erhalten werden. Anders bereitete Goldsalzlösungen erzeugten an den eingetauchten Fasern nur Dunkelfärbung.

\section{Ausführung der Reaktion.}

Die Reaktion wurde ausgeführt, wie sie Professor Emich für seine Lackmusseide ${ }^{1}$ angibt. Ein gebrauchsfertiger Kokonfaden wurde an einem Wachsklötzchen befestigt, mit einer Scheere so abgeschnitten, daß ein etwa zentimeterlanger Teil frei blieb und zur Feststellung seiner tadellosen Beschaffenheit im Mikroskop betrachtet. Von der fraglichen Flüssigkeit wurde ein Tröpfchen auf eine passende Unterlage gebracht und hierauf das Ende des Kokonfadens lotrecht eingetaucht, damit es während der Verdunstung der Wirkung der sich konzentrierenden Lösung ausgesetzt war. Endlich wurde die Faser unter dem Mikroskop geprüft, wobei Kondensorbeleuchtung und 200 fache Vergrößerung zur Anwendung kamen.

\section{Empfindlichkeit der Reaktion.}

Um eine Vorstellung über die Empfindlichkeit dieser Reaktion zu gewinnen, wurden zwei Versuchsreihen angestellt. Die eine (a) verfolgte den Zweck, den Verdünnungsgrad einer Goldlösung festzustellen, bei welcher eine in dieselbe getauchte Faser noch eine charakteristische Goldfärbung annahm; in der anderen Versuchsreihe (b) wurde ermittelt, welche kleinste Menge jener Lösungen an dem Fadenende eben noch die erwähnten Färbungen zu stande brachte. Daraus ergab sich die kleinste absolute Menge nachweisbaren Goldes. 
a) Baumwolle oder Seide, die mit Tannin behandeit worden waren, wurden in Goldlösungen, die 0.07 (Lösung I), beziehungsweise $0 \cdot 04 \mathrm{mg}$ (Lösung II) pro $\mathrm{cm}^{3}$ enthielten, innerhalb 3 Minuten dunkel gefärbt. Verdünntere Lösungen färbten die Fäden etwas langsamer; doch kamen diese Verdünnungen hier nicht in Betracht, weil die in der zweiten Versuchsreihe angewandten Flüssigkeitsmengen so gering waren, daß sie in kürzester Zeit verdunsteten.

Für Wolle und Seide, die im Lösungsgemisch von Pyrogallol und Zinnchlorür gelegen hatten, genügte ein Goldgehalt von 0.016 (Lösung III), beziehungsweise $0.008 \mathrm{mg}$ (Lösung IV), um sie zu färben. Auch hier wurde aus demselben Grunde wie oben von größeren Verdünnungen abgesehen.

b) Die kleinsten, zur Reaktion eben noch hinreichenden Mengen jener Lösungen wurden mittels einer kapillaren Pipette gemessen und betrugen für die einzelnen Fälle:

1. $0 \cdot 00048 \mathrm{~cm}^{3}$ der Lösung I für Baumwolle, gebeizt in Tannin.

2. $0 \cdot 00036 \mathrm{~cm}^{3}$ der Lösung II für Seide, gebeizt in Tannin;

3. $0.00120 \mathrm{~cm}^{3}$ der Lösung III für Baumwolle, gebeizt in Pyrogallol und Zinnchlorür.

4. $0 \cdot 00024 \mathrm{~cm}^{3}$ der Lösung IV für Seide, gebeizt in Pyrogallol und Zinnchlorür.

Daraus ergaben sich folgende Werte für die kleinsten absoluten Mengen nachweisbaren Goldes:

a) $0.032 \mu g^{-1}$ für Baumwolle, in Tannin gebeizt;

b) $0.015 \mu g$ für Seide, in Tannin gebeizt;

c) $0.020 \mu g$ für Baumwolle, in Zinnchlorür und Pyrogallol gebeizt;

d) $0.002 \mu g$ für Seide, in Zinnchlorür und Pyrogallol gebeizt.

Vergleicht man damit die Empfindlichkeit der bis jetzt bekannten mikrochemischen Goldreaktionen, ${ }^{2}$ so findet man sie durch den Fall $d$ um das Tausendfache übertroffen. Es ist daher die im Zinnchlorür

$11 \mu g=0.001 \mathrm{mg}$.

2 Behrens, Mikrochemische Analyse, 2. Aufl., p. 80. 
und Pyrogallol gebeizte Seidenfaser besonders geeignet, um kleine Mengen von Gold nachzuweisen.

\section{Störungen der Reaktion.}

Die bisher mitgeteilten Versuche gingen stets von reinen Goldlösungen aus. Die Untersuchungen über den Einfluß anderer Substanzen auf die Reaktion haben ergeben, daß es verhältnismäßig wenig Stoffe gibt, bei deren Gegenwart die Färbung der Fasern Einbuße erleidet oder ganz ausbleibt.

Von vornherein ausgeschlossen ist selbstverständlich die Anwesenheit freier Halogene oder anderer Oxydationsmittel, sowie die reduzierend wirkenden Substanzen.

Von den vielen ausgeführten Versuchen kommen im folgenden nur diejenigen in Betracht, die mit den in Zinnchlorür und Pyrogallol gebeizten Seidenfäden ausgeführt wurden. Die dabei angewendete Goldlösung enthielt $0.03 \mathrm{mg}$ Gold im $\mathrm{cm}^{3}$. Zunächst wurde der Einfluß der Alkalimetalle untersucht. Es zeigte sich dabei, daß Alkalisaize mit Ausnahme der Silikate die Reaktionen nicht beeinflussen. So wurde bei Anwesenheit von selbst sehr großen Kochsalzmengen der Faden doch rot, nur war der Farbton gegenüber der Färbung in der reinen Goldlösung etwas verändert. Gewöhnlich erschien der Faden ziegelrot bis zimmtbraun. Hier möge erwähnt werden, daß sich der Baumwollfaden, der analog der Seidenfaser vorbehandelt wurde, bei Gegenwart von Kochsalz gar nicht färbte; wohl aber färbte sich der mit Tanninlösung behandelte Baumwollfaden in schwach alkalischer, Alkalisalze enthaltender Goldlösung schön blau. Alkalibasen, und das gilt auch von den anderen Basen, verhindern die Färbung des Seidenfadens vollständig.

Magnesium und die Erdalkalimetalle sind ohne besonderen Einfluß auf die Färbung, nur scheint sie mehr ins Fleischrote zu spielen.

Aluminium- und Chromsalze beeinflussen die Färbung nicht.

Zink,- M angan-, Nickel- und Kobaltsal ze desgleichen; die letzteren scheinen den Faden lebhafter rotwerden zu lassen. 
Eine Ausnahme machen die Eisenoxydsalze, welche, offenbar wegen ihrer oxydierenden Eigenschaften, die Rotfärbung verhindern. In genau neutralisierter Lösung gelang es, den Faden zu färben, doch wurde derselbe nur blaugrau, seltener braunrot.

Beim Silber wurde die merkwürdige Beobachtung gemacht, daß seine Salze allein schon fähig sind, den Faden zu färben. Dieser erschien, im Mikroskope betrachtet, strohgelb, dunkler oder lichter, je nach der Konzentration. Beim Behandeln mit Salpetersäure verschwand die Gelbfärbung sofort. Der Einfluß der Silbersalze auf die Goldreaktion des Fadens kommt aber nicht in Betracht, weil die gelbe Farbe von der roten verdeckt wird, und weil sich andrerseits eine zweifelhafte schwache Färbung durch Behandeln mit Salpetersäure leicht näher charakterisiert.

Von den Quecksilbersalzen wurde das Sublimat und das Oxydnitrat geprüft, doch ergab sich auch hier keinerlei Störung der Goldreaktion.

Blei-, Wismut- und Kupfersalze zeigten sich ebenfalls indifferent.

Bei Gegenwart von Kadmium wurde der Faden bläulichrot,

Arsen in der Form von arseniger Säure oder Arsensäure verhinderte natürlich die Färbung vollständig; ebenso Brechweinstein und Antimoniate.

Platinsalzlösungen veränderten den eingetauchten präparierten Faden gar nicht und verhinderten auch die Goldreaktion nicht, sofern sie nicht in großem Überschuß da waren, in welchem Falle der rote Seidenfaden sehr bald geschwärzt wurde.

Von den in Betracht kommenden Säuren verhinderte die Kieselsäure die Färbung der Faser. Das Gleiche war natürlich auch bei Gegenwart konzentrierter Mineralsäuren der Fall.

\section{Anwendung der Reaktion.}

Diese Reaktion scheint namentlich dort gut anwendbar $z \mathrm{u}$ sein, wo es sich um den Nachweis geringer Mengen Goldes 
neben großen Massen anderer Stoffe handelt. Wie man hiebei verfahren kann, ist im folgenden kurz beschrieben.

a) Das goldführende Gemenge wurde mit Königswasser ausgezogen, die Lösung eingedampft und der Rest in Wasser aufgenommen. Die Flüssigkeit wurde mit etwa $1 \mathrm{cg}$ Kobaltsalz versetzt, mit doppeltkohlensaurem Natrium neutralisiert und mit Essigsäure angesäuert. Hierauf wurden Kobalt und Gold mit einem Überschusse von salpetrigsaurem Kali gefällt, der Niederschlag wurde ausgewaschen, in Königswasser gelöst, die Lösung eingedampft, der Rest in Wasser aufgenommen und auf Gold geprüft. Nach dieser Methode wurden ein brasilianischer Flußsand, Schwefelkies aus Schelgaden, ein Siebenbürger goldhaltiges Erz u. a. mit Erfolg auf Gold geprüft. Doch stellten sich bei diesem Verfahren bald verschiedene Nachteile ein. Abgesehen davon, daß in die Endlösung fast immer Eisen mitgeschleppt wurde, das die Reaktion, wie bereits erwähnt, beeinträchtigt, konnte auch die Konzentration der Flüssigkeit infolge des auskristallisierenden Kobaltsalzes nicht weit genug getrieben werden.

b) Eine andere Methode bestand darin, daß man die goldhältige Lösung, die mit Schwefelsäure angesäuert wurde, mit einem Gemisch von Kaliumnitrit und Baryumchlorid versetzte. Das goldhältige Baryumsulfat wurde mit Königswasser behandelt, das Gold nach dem Eindampfen mit Wasser aufgenommen und auf die bekannte Weise nachgewiesen. Diese Methode war zum Teil mit denselben Nachteilen behaftet wie die oben erwähnte.

c) Am besten hat sich bis jetzt folgendes Verfahren bewährt: Die auf Gold zu untersuchende, von freiem Chlor befreite Lösung wurde mit Bleifeile versetzt und langsam fast bis zur Trocknis eingedampft. Nun wurde mit Wasser verdünnt, der Bodensatz durch Dekantieren ausgewaschen und mit Königswasser behandelt; aus der Lösung wurde die kleine Menge gelösten Bleies mittels verdünnter Schwefelsäure gefällt. Das chlorhältige Filtrat wurde zuerst am Wasserbade, schließlich am Sandbade zur Vertreibung der Schwefelsäure eingedampft und der Rückstand wie oben behandelt. Nach dieser Methode wurden beispielsweise wiederho!t Gemische von 5 bis $10 g$ 
Metalloxyd (z. B. Eisen- und Kupferoxyd) mit Sand geprüft, welchen der zehnmillionste Teil ihres Gewichtes an Gold zugesetzt worden war; letzteres konnte in der Endlösung stets nachgewiesen werden. Auch viele Kiese und andere Mineralien wurden bereits auf diese Art untersucht. Hier muß noch bemerkt werden, daß in einigen verwickelten Fällen statt der Rotfärbung des Seidenfadens eine blaue bis blaugraue Farbe beobachtet wurde; doch ließ sich diese Färbung durch ihre Beständigkeit gegenüber Säuren und Basen sehr leicht als eine Goldreaktion erkennen, auch geht sie beim Erwärmen in der Regel in die rote über.

Weitere Versuche, welche namentlich den Zweck haben Gold in gefärbten Mineralien aufzusuchen, sind im Gange Vielleicht gelingt es auch den Nachweis zu erbringen, daß einige von ihnen ihre Farbe kolloidal gelöstem Golde verdanken. 